\title{
A Telepresence Robotic System operated with a P300-based Brain-Computer Interface: Initial Tests with ALS patients
}

\author{
Carlos Escolano, Ander Ramos Murguialday, Tamara Matuz, Niels Birbaumer, and Javier Minguez
}

\begin{abstract}
Brain-computer interfaces (BCIs) open a new valuable communication channel for people with severe neurological or motor degenerative diseases, such as ALS patients. On the other hand, the ability to teleoperate robots in a remote scenario provides a physical entity embodied in a real environment ready to perceive, explore, and interact. The combination of both functionalities provides a system with benefits for ALS patients in the context of neurorehabilitation or maintainment of the neural activity. This paper reports a BCI telepresence system which offers navigation, exploration and bidirectional communication, only controlled by brain activity; and an initial study of applicability with ALS patients. The results show the feasibility of this technology in real patients.
\end{abstract}

\section{INTRODUCTION}

Brain-computer interfaces (BCIs) provide their users communication and control with their brain activity alone. They do not rely on the brain's normal output channels of peripheral nerves and muscles, opening a new valuable communication channel for people with severe neurological or muscular diseases. The great advances in the BCIs and robotics interaction have made possible to use the brain electrical activity online to control robotic devices with an augmentative or restoration function.

A population that could benefit from BCI technologies is patients with amyotrophic lateral sclerosis (ALS). ALS is a progressive neurological degenerative disease that leads to the locked-in syndrome (LIS), which is characterized by complete motor paralysis, except for one muscle used for communication, with intact cognition and sensation [1]. The ability to brain-teleoperate robots in a remote scenario opens a new dimension of possibilities for patients with severe neuromuscular disabilities. It provides them with a physical entity embodied in a real environment (anywhere in the world with Internet access) ready to perceive, explore, and interact, and controlled only by brain activity. In fact, it has been suggested that the engagement of patients in using such BCIs could elicit a neurorehabilitation effect and/or a maintainment of the neural activity avoiding or delaying

Carlos Escolano and Javier Minguez are with the Instituto de Investigación en Ingeniería de Aragón (I3A) and Dpto. de Informática e Ingeniería de Sistemas (DIIS), Universidad de Zaragoza, Spain. E-mail: \{cescolan, jminguez\}@unizar.es. Ander Ramos Murguialday, Tamara Matuz and Niels Birbaumer are with the Institute of Medical Psychology and Behavioral Neurobiology, Tubingen, Germany. Email: ander.ramos@fatronik.com, tamara.matuz@medizin.uni-tuebingen.de, niels.birbaumer@uni-tuebingen.de. Ander Ramos Murguialday is also with Fatronik Tecnalia Germany, Tubingen, Germany. Niels Birbaumer is also with the Ospedale San Camilo-IRCCS, Istituto di Ricovero e Cura a Carattere Scientifico, Venezia, Lido, Italy. This work has been partially supported by projects HYPER-CSD2009-00067, DPI2009-14732-C02-01 funded by the Spanish Government.

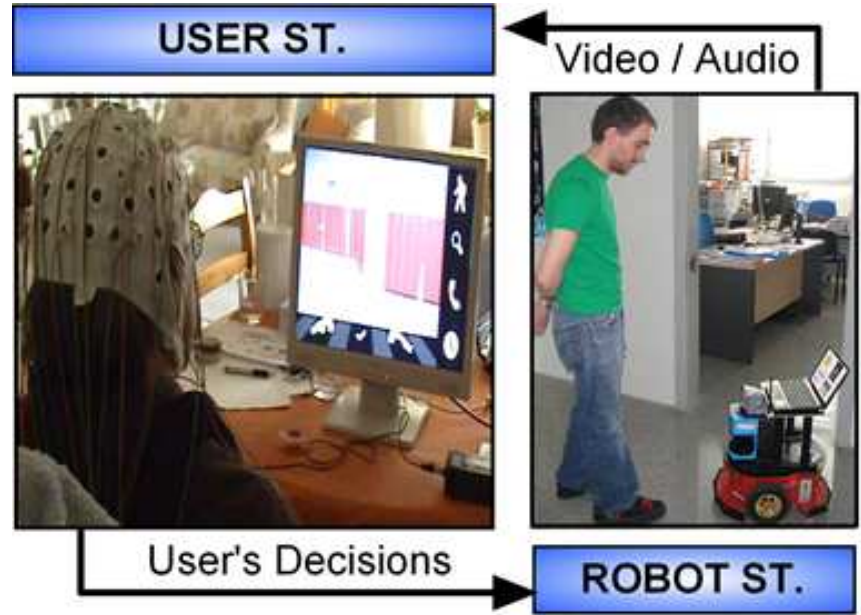

Fig. 1. ALS patient operates with a BCI the robotic telepresence system.

this way the extinction of thought, hypothesized to happen in ALS patients [1]. A commonly used brain signal in the development of communication BCIs for ALS patients is the P300 event-related potential [2].

In this direction, a P300-based brain-controlled teleoperation system of a mobile robot with navigation and exploration capabilities was already developed [3]. Furthermore, that study explored the applicability of this technology with healthy users with satisfactory results. The results showed the need to improve the interaction capabilities to address the real patients needs and performance restrictions. The present system improves the previous functionalities with a bidirectional communication of video and audio, and user interaction (the user can send preconfigurable sentences, binary responses or alarms). All these changes were designed following patients, caregivers and family suggestions to improve communication in LIS patients. The commands and alarms were adjusted to common needs incorporating a novel interaction mode. This paper reports an initial study of applicability of this new design with ALS patients. Furthermore, this is the first time that a brain-controlled telepresence system has been used by an ALS patient.

\section{BCI AND ROBOTIC DEVICE}

The telepresence system is composed by a user station (patient environment) and a robot station (placed anywhere in the world), both remotely located and connected via Internet (Figure 1). The underlying idea of the system is that in the user station the brain-computer system decodes the user's intentions, which are transferred to the robotic system via 


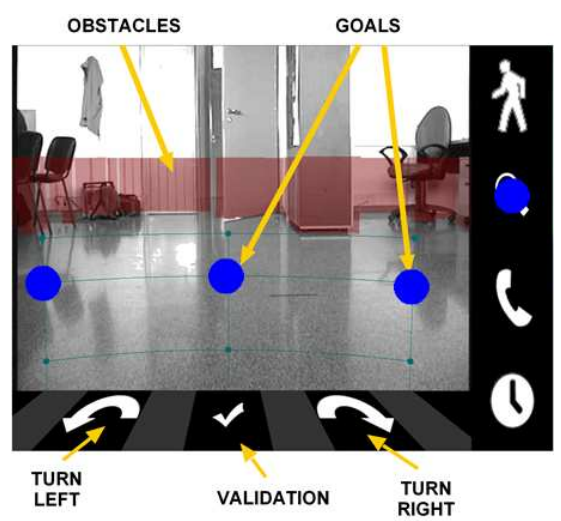

(a) Navigation interface

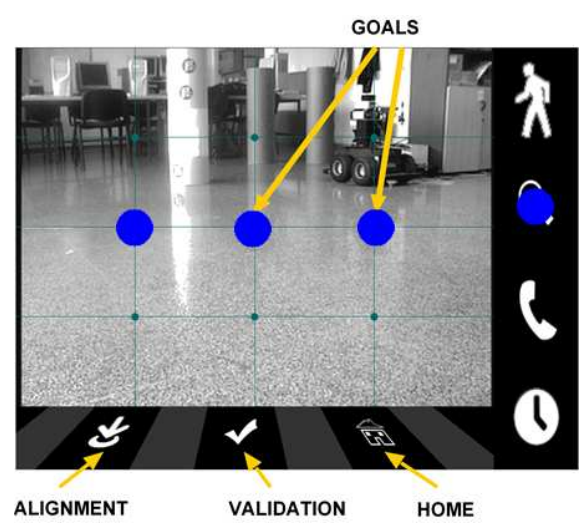

(b) Exploration interface

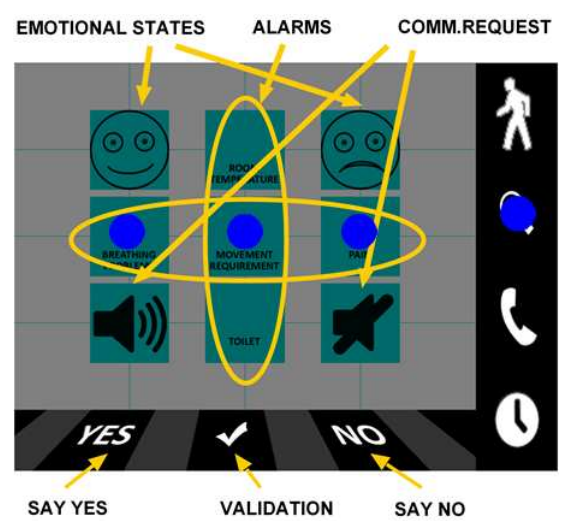

(c) Interaction interface

Fig. 2. The BCI graphical interfaces of each operation mode. The three graphical interfaces have the four column in common, which allows changing the operation mode. The last option in the four column allows pausing the system (i.e. stopping the P300 stimulation process) for a configurable amount of time, and receiving video and audio from the remote environment. The options are stimulated (flashed) by means of rows and columns displaying a blue circle on them. An example of a flashing of the second row options is shown for each interface.

Internet. Furthermore, the robotic system sends live video and audio (captured by the robot camera and microphone), which are used by the user as feedback for decision-making and process control. In operation, the user can alternate between three operation modes: $(i)$ robot navigation mode, (ii) camera exploration mode, and (iii) interaction mode. In all modes, the user faces a screen with different options according to the operation mode (Figure 2), which are arranged in a $4 \times 4$ matrix to favor the next P300 oddball paradigm.

In the navigation mode the visual display shows an augmented reality reconstruction of the robot station. In this reconstruction the obstacles are depicted as 3D semitransparent walls built from a 2D map constructed in real-time by the autonomous navigation technology integrated in the robot. Over that representation, the users can select a location of the space using the BCI to order the robot to move there (unreachable destinations could not be selected since they will be hidden by the 3D walls, helping the user to avoid confusions and improving the robustness of the system). Once a location is selected, it is transferred to the navigation technology [4], which drives the robot avoiding collisions with the obstacles (both static and dynamic) detected by its laser scanner. Notice that this kind of strategy allows to safely navigate in unknown and populated scenarios, which is one of the most challenging issues of telepresence if we want to support the possibility of a patient teleoperating a robot in any social activity. In the exploration mode the visual display shows a $2 \mathrm{D}$ grid uniformly arranged in the display mapping a predefined set of locations that the user can select to orientate the camera. Thus, it provides the users with active visual exploration capabilities. In the interaction mode the visual display shows a 2D set of options that the user can select to communicate with the remote scenario, such as five primary alarms (to express breathing problems, movement requirement, pain, inadequate room temperature, toilet need), two emotional states (feels happy or sad), two

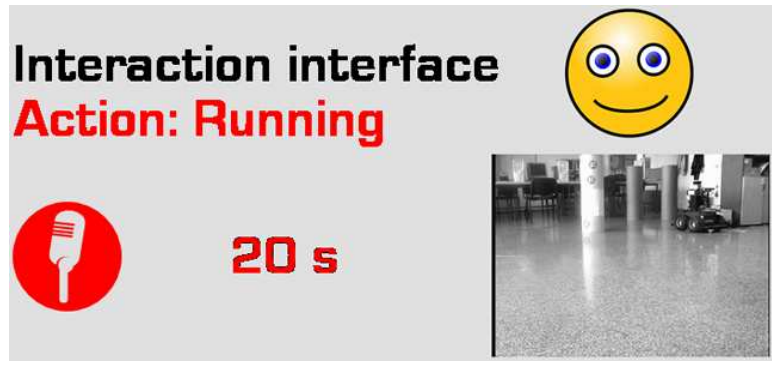

Fig. 3. Robot station graphical interface, displayed in the laptop placed on the robot. This interface shows the actions that the user is performing, his emotional state, and whether video and audio is being sent to the user. Furthermore, when the robotic device is sending video and audio, it displays that video and the resting time to stop the transfer.

binary responses (yes, no), and two options to express the willingness to establish or finish a communication with anybody in the vicinity of the robot. In order to provide the people in the vicinity of the robot with a feedback of the user's decisions while teleoperating the robot, the user selected actions are displayed in the graphical interface of the laptop placed on the robot (Figure 3).

The execution protocol is modeled as a finite-state machine: $(i)$ the BCI graphical interface develops a stimulation process (flashing) over all the possible options following the P300 oddball paradigm; (ii) the signal processing strategy detects the target the user is concentrated on; (iii) once the desired target is selected, the user must subsequently select the validation option to send the target to the robotic system (this redundancy minimizes the probability of sending incorrect orders to the robotic system although BCI errors happen); (iv) the robotic system executes the order (this will be referred as a mission); $(v)$ while the mission is being performed in navigation and exploration modes the robot sends live video and audio, in the interaction mode video and audio are sent for 30 seconds in order to allow short periods for a binary conversation. This time was empirically proved to be enough for a simple but successful communication. 


\section{METHODS}

\section{A. Participants}

A 54 years old individual suffering from amyotrophic lateral sclerosis (ALS) participated in the study. The first diagnosis was performed on January 2006 resulting in a diagnosis of a sporadic spinal ALS. At the time of the telepresence experience the individual was classified with an ALS functional rating score (ALS-FRS) [5] of 15. The study was approved by the Ethical Review Board of the Medical Faculty of the University of Tubingen in Germany. The telepresence experience was performed being the patient in his home (South Germany) and the brain teleoperated robot in the University of Zaragoza (Spain).

\section{B. Data acquisition}

EEG data was recorded using a commercial gTec EEG system (two gUSBamp amplifiers). 24 EEG electrodes were placed in the locations FP1, FP2, F3, F4, C3, C4, P3, P4, T7, T8, CP3, CP4, Fz, Pz, Cz, OZ, FC3, FC4, F7, F8, $\mathrm{P} 7, \mathrm{P} 8, \mathrm{FCz}$ and $\mathrm{CPz}$ according to the international $10 / 20$ system. The ground electrode was positioned on the forehead (position $\mathrm{Fz}$ ) and the reference electrode was placed on the left earlobe. The EEG was amplified, digitalized with a sampling frequency of $256 \mathrm{~Hz}$, power-line notch filtered and bandpass-filtered between 0.5 and $30 \mathrm{~Hz}$. The signal recording and processing, as well as the graphical interface, were developed under BCI2000 platform [6], placed on an Intel Core2 Duo @ 2.10GHz with Windows XP OS.

\section{Signal Processing}

A two-step supervised learning technique was used: $(i)$ feature extraction, and $(i i)$ classification algorithm. In order to extract the features, one-second sample recordings were extracted after each stimulus onset for each EEG channel. These segments of data were then filtered using the moving average technique and downsampled by a factor of 16 . The resulting signals were plotted and the channels with the best P300 response were selected by visual inspection. The resulting data segments for each channel were concatenated, creating a single-feature vector for the classification algorithm. Since the P300 oddball paradigm was followed to reduce the duration of a sequence and the dimension of the pattern-recognition problem, P300 signal was elicited for one of the rows or columns during the sequence of stimulation, obtaining two classification problems of 4 classes. For each of these subproblems the StepWise Linear Discriminant Analysis (SWLDA) was used, extensively studied for P300 classification problems [7].

\section{Study Design}

The study was conducted in two different sessions (4 and 13 November, 2009) with the same participant. It was divided in three phases: $(i)$ a screening and training phase, (ii) an online phase to perform a goal-oriented telepresence predefined task, and $(i i i)$ an online phase to freely explore all the functionalities of the telepresence system. The objectives of the study were to evaluate whether the P300 response was

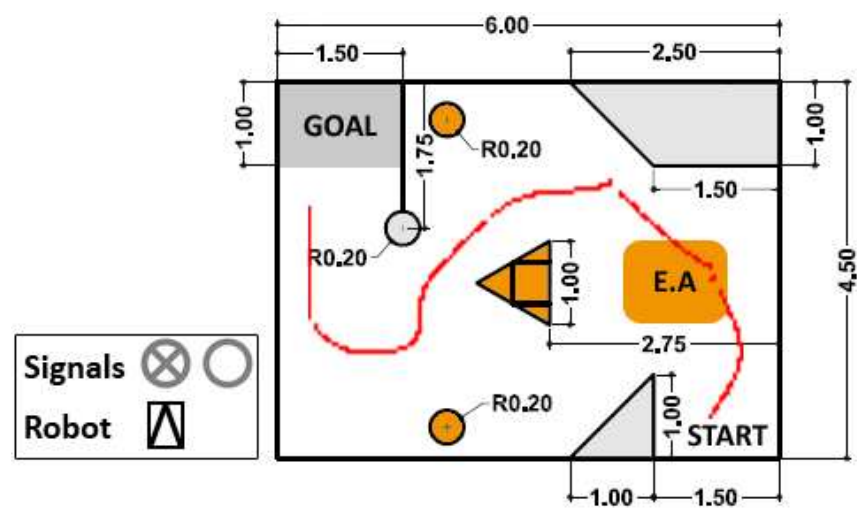

Fig. 4. The objective of the task was to drive the robot from the start location to the goal area. In the exploration area (E.A. in the figure), the patient had to look for two yellow cylinders, in which a sign $2.5 \mathrm{~m}$ above the floor on each cylinder was placed. Then, if both signals were equal, the patient had to avoid the yellow triangle by turning to the right-hand side, or if otherwise, by turning to the left-hand side. Red line shows the real trajectory of the patient in the first trial of the second session.

elicited in ALS patients by the graphical interface of the system, to measure whether it can be detected with a minimum of $70 \%$ accuracy (suggested as a predictor for satisfactory communication [1]), and to explore the boundaries of the telepresence system and its real usefulness for ALS patients. The tasks, procedures and objectives of each phase are next detailed.

\section{E. Tasks and Procedures: Phase I}

This phase was composed by two tasks: $(i)$ a screening task to study the P300 response, and (ii) a training task to calibrate the system and evaluate the online BCI accuracy. In these tasks the participant had to attend a predefined set of targets in the graphical interface. The number of sequences and all the scheduling of the stimulation process, mainly the inter-stimulus interval (ISI) and stimulus duration, were customized for each task.

In the screening task the participant had to attend to 3 targets. The number of sequences was set to 5 , the ISI to 1 sec (to avoid the P300 overlapping) and the stimulus duration to $125 \mathrm{~ms}$. In the training task the participant had to perform 4 training trials to calibrate the system and online trials to evaluate whether he was able to achieve a minimum of $70 \%$ accuracy. In each trial, a sequence of 8 targets had to be attended (the even targets in 2 trials and the odd ones in the other 2 trials to cover all the row and columns). The number of sequences was set to 10 , the ISI to $75 \mathrm{~ms}$ and the stimulus duration time to $125 \mathrm{~ms}$. The complete phase lasted $25 \mathrm{~min}$.

\section{F. Tasks and Procedures: Phase II}

The objective of the phase was to evaluate the online BCI accuracy, the navigation and exploration capabilities of the system, its usefulness and its easy of use in a goaldirected predefined task. The participant had to accomplish two trials of a complex task that jointly involved navigation in constrained spaces and the active search of two visual targets (Figure 4). The number of sequences and all the 


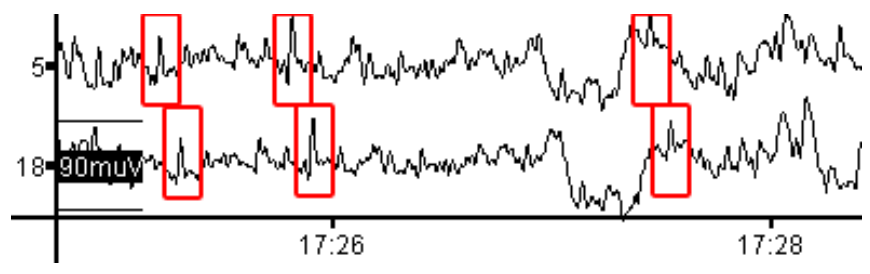

Fig. 5. EEG desynchronization example between a channel of the first amplifier (channels 1-16) and a channel of the second one (channels 1724).

scheduling of the stimulation process was set to the same values as the training task in Phase I. The study was accomplished between the patient's home (South Germany) and the University of Zaragoza (Spain), where the robot was placed, both connected via Internet. The only information of the remote scenarios shown to the patient prior to the experiment was the plan referenced above. Note that the same task was performed successfully by five healthy users in a previous study [3], although in this paper we do not intend to compare healthy versus ALS affected individual performance. After this phase, the participant was presented with a battery of neuropsychological questionaries like the Questionnaire for Current Motivation (QCM) and Skalen zur Erfassung der Lebensqualität (SEL, engl.: Scales for the assessment of quality of life), described in the section 2.4 from [8], to study motivation and mood. A cognitive assessment form was used to analyze his feelings using the device during the task. This entire phase consisting on telepresence experience and questionaries lasted about 1.5 hours.

\section{G. Tasks and Procedures: Phase III}

The objective of the phase was to evaluate the usefulness of the overall telepresence and communication system, focusing more on the interaction mode. The participant had to freely use the system functionalities for at least 25 minutes with the only requirement of using the interaction interface to communicate with any of the BCI team researchers in the University of Zaragoza at least once. The number of sequences and all the scheduling of the stimulation process was set to the same values as the training task in Phase I. After this phase, the participant was presented with the same battery of neuropsychological questionaries of Phase II.

\section{RESUlTS}

\section{A. Phase I}

Phase I was successfully completed in the two sessions. Firstly, the participant performed the designed screening task and it was found by visual inspection of the EEG data recorded, that the P300 potential was elicited (in both sessions) at a latency of roughly $400 \mathrm{~ms}$ in the central-parietal and occipital lobes. Secondly, the participant performed the training task. This training or calibration task lasted only about 10 minutes. The signal processing strategy was applied to the collected EEG data and a $100 \%$ theoretical accuracy was thrown by the classifier. After that, the classifier results
TABLE I

METRics TO EVAluATE THE TELEPRESENCE SySTEM PERFORMANCE

Table show the results of some metrics in the three trials performed in Phase II. The metrics are: task success, the BCI accuracy; the time, path length and number of missions used to complete the task; and the ITR. Note that missions have been defined in section II as a order sent to the robotic device. There is a distinction between real/estimated in some metrics to distinguish the obtained results in the online trials and the estimated results after removing the artifact.

\begin{tabular}{l|c|c|c}
\hline & Session 1 & \multicolumn{2}{|c}{ Session 2 } \\
& Trial 1 & Trial 1 & Trial 2 \\
\hline Task success & 1 & 1 & 1 \\
Real BCI accuracy & $57 \%$ & $44 \%$ & $38 \%$ \\
Estimated BCI accuracy & $84 \%$ & $81 \%$ & $70 \%$ \\
Real time (sec) & 1884 & 2021 & 2277 \\
Estimated time (sec) & 1372 & 910 & 975 \\
Path length (m) & 10.99 & 13.53 & 11.84 \\
\# missions & 19 & 15 & 11 \\
Estimated ITR (bits/min) & 8.22 & 7.67 & 5.84 \\
\hline
\end{tabular}

were tested in an online trial to evaluate the real accuracy. The participant achieved a $100 \%$ and a $90 \%$ in both sessions respectively over a 10 targets trial. In summary, the designed graphical interface and stimulation process were able to elicit the P300 response and it could be detected with a higher accuracy than the $70 \%$ defined threshold value in BCI control for satisfactory communication.

\section{B. Phase II}

The participant succeeded in solving the task jointly combining navigation and exploration using the robot. In the first session, just trial 1 was performed (due to participant tiredness caused in part by some hardware and software issues) and in the second one the two trials were performed. Before addressing the participant performance, we would like to report there was an artifact in the EEG signal that was detected in the posterior data analysis but affected the BCI online performance: the two amplifiers desynchronized due to software overload with Internet delays (Figure 5). The data has been offline processed and the artifact removed. Table I show the results with/without the artifact.

The teleoperation task was successfully solved, thereby we conclude that the options provided by the graphical interface were sufficient and practical. The number of sequences and all the scheduling of the stimulation process established the number of selections per minute to 3 . The BCI accuracy was low due to the software artifact, which caused several incorrect selections (on average 46\%). The elimination of the artifact from the analysis (which affects to $55 \%$ of the total number of selections) turned the BCI accuracy to $78 \%$. Considering the estimated BCI accuracy, the average information transfer rate (ITR) according to the Wolpaw definition [9] ${ }^{1}$ is $7 \mathrm{bits} / \mathrm{min}$, being on the range of typical P300-based systems. Concerning the robot navigation, no

\footnotetext{
${ }^{1} B=\log _{2} N+P \log _{2} P+(1-P) \log _{2} \frac{1-P}{N-1}$ where B is the number of bits per trial (i.e. bits per selection), $\mathrm{N}$ is the number of possible selections, and $\mathrm{P}$ is the probability that a desired selection will occur.
} 
collisions were reported. The real time to complete the task was long due to the low BCI accuracy, although the estimated time is acceptable for such a complex task and is in the order of magnitude of the previous study with healthy individuals [3]. Path length is quite similar among trials due to the execution of a task in a very constrained space. The number of missions varied among trials, suggesting that the participant used different strategies to complete the task. Furthermore, the number of missions decreased among trials, which suggests that the user learned to solve the task more efficiently.

These results are very encouraging since they show the feasibility of the technology helping ALS patients to solve tasks in which jointly navigation and visual exploration are needed, in unknown scenarios and real settings. Furthermore motivation and mood increased after the second session, reflected in the QCM and SEL scales. This could indicate that although the system was slow and tiring the patient was engaged and motivated by the task.

\section{Phase III}

This phase was only performed in the second session. In this phase the participant freely controlled the brainactuated telepresence system for 25 minutes. In that time, he was able to perform an exploration of the environment and establish a communication with a member of the BCI team in the University of Zaragoza: this member asked some yes/no questions that could be answered by the patient with the options provided in the interaction graphical interface. The participant found very useful the interaction interface and reported excitement towards more sessions and use of the telepresence device. A clear positive and satisfactory evaluation was obtained from the usability and usefulness questionaries handed out to the patient after the sessions.

\section{Conclusions}

This paper presents an evaluation of a P300-based brainactuated robotic telepresence system by an ALS patient. This system incorporates advanced autonomous navigation, active visual exploration, and communication capabilities. From a navigational point of view, the great advantage is that the user selects destinations from a set of generated points in the environment that can be autonomously and safely reached. From an interactional point of view, despite the low ITR of P300-based systems there are two advantages. The first advantage is that once the order is given to the system the user can relax until the next decision needs to be made. Since the functionalities are automated our system avoids the exhausting mental processes of other devices. The second advantage is that the command information has to travel along the Internet but it is autonomously executed by the robot, avoiding the information transfer delay problem of teleoperation systems with continuous control.

The evaluation results are encouraging since they show the feasibility of using this technology in patients with severe neuromuscular disabilities. In this work we tackled 3 issues concerning communication wit LIS patients: $(i)$ short alarms can be selected for a prompt reaction of the caregiver or whoever is in the vicinity of the telepresence controlled robot; $(i i)$ binary communication can be established any time offering a telecommunication possibility; and (iii) the system can be paused if a resting time or a pause time is needed. Furthermore, a spatial navigation and visual exploration can be achieved allowing the patient to explore remote scenarios. All this together depicts our telepresence system as an interesting, working, enjoyable and attractive system. Not only has a use as a remote presence device but can provide also a more joyable way of brain training (P300 based in our case). Furthermore it has been suggested that the engagement of the patient in this kind of systems could produce a neurorehabilitation effect, maintaining the neural activity related to spatial navigation, action and communication, avoiding or delaying this way the extinction of thought in late stages ALS patients [1]. As synchronization software artifacts occurred, researchers are working in a more robust system to accomplish new tests with more ALS patients.

\section{ACKNOWLEDGEMENTS}

Thanks are extended to the BCI team in the University of Zaragoza and to the Institute of Medical Psychology and Behavioral Neurobiology of Tubingen for their support, and specially to the participant of the study.

\section{REFERENCES}

[1] A. Kübler and N. Birbaumer, "Brain-computer interfaces and communication in paralysis: Extinction of goal directed thinking in completely paralysed patients?," Clinical Neurophysiology, vol. 119, no. 11, pp. 2658 - 2666, 2008.

[2] S. Sutton, M. Braren, J. Zublin, and E. R. John, "Evoked potential correlates of stimulus uncertainty," Science, vol. 150, no. 3700, pp. 1187-1188, 1965.

[3] C. Escolano, J. Antelis, and J. Minguez, "Human Brain-Teleoperated Robot between Remote Places," IEEE International Conference on Robotics and Automation (ICRA), 2009.

[4] L. Montesano, J. Minguez, and L. Montano, "Lessons learned in integration for sensor-based robot navigation systems," International Journal of Advanced Robotic Systems, vol. 3, no. 1, pp. 85-91, 2006.

[5] Jesse M. Cedarbaum and Nancy Stambler, "Performance of the Amyotrophic Lateral Sclerosis Functional Rating Scale (ALSFRS) in multicenter clinical trials," Journal of the Neurological Sciences, vol. 152, no. Supplement 1, pp. s1 - s9, 1997.

[6] G. Schalk, D.J. McFarland, T. Hinterberger, N. Birbaumer, and J.R. Wolpaw, "BCI2000: A General-Purpose Brain-Computer Interface (BCI) System," IEEE Transactions on Biomedical Engineering, vol. 51, no. 6, May 2004.

[7] L.A. Farwell and E. Donchin, "Talking off the top of your head: toward a mental prosthesis utilizing event-related brain potentials," $E E G$ Clinical Neurophysiology, vol. 70, no. 6, pp. 510-23, 1988.

[8] S.C. Kleih, F. Nijboer, S. Halder, and A. Kübler, "Motivation modulates the P300 amplitude during brain-computer interface use," Clinical Neurophysiology, vol. In Press, 2010.

[9] J. R. Wolpaw, N. Birbaumer, W. J. Heetderks, D. J. McFarland, P. H. Peckham, G. Schalk, E. Donchin, L. A. Quatrano, C. J. Robinson, and T. M. Vaughan, "Brain-computer interface technology: a review of the first international meeting.," IEEE Trans Rehabil Eng, vol. 8, no. 2, pp. 164-173, June 2000. 